\title{
Static structural health monitoring and automated data analysis procedures applied to the diagnosis of a complex medieval masonry monastery
}

\author{
Nirvan Makoond ${ }^{\mathrm{a}}$, Luca Pelàa ${ }^{\mathrm{a}}$, Climent Molins ${ }^{\mathrm{a}}$, and Pere Roca ${ }^{\mathrm{a}}$ \\ ${ }^{a}$ Department of Civil and Environmental Engineering, Universitat Politècnica de Catalunya \\ (UPC-BarcelonaTech), Jordi Girona 1-3, 08034 Barcelona, Spain
}

\begin{abstract}
Static structural health monitoring (SHM), aimed at the continuous measurement of slow-varying parameters over a long period, has been proved to be a powerful tool to support the diagnosis of masonry heritage structures. In such applications, the initial interpretation task involves the identification of evolutionary conditions from recorded data. However, this can be difficult since monitored features are influenced by environmental changes. In addition, many masonry heritage structures are characterised by a complex structural behaviour stemming from the interaction among different elements, making the task of interpreting SHM data for diagnosis very challenging. One such structure is the church of the monastery of Sant Cugat close to Barcelona, built mostly between the 12th and 15th centuries. Certain key structural parameters of the church have been monitored since 2017 with the aim of understanding the cause of visible pathologies and identifying any active deterioration mechanisms that could pose a threat to the structural integrity of the church in the future. This paper presents the application of an automated data analysis methodology to this problem. The method uses dynamic regression models to filter out components related to reversible seasonal fluctuations from measurements and automatically classifies monitored parameters into evolutionary states based on predicted evolution rates and dispersion metrics from the filtering procedure. A tool is presented which allows analysis results to be updated as new data is received. Finally, results from the proposed methodology are used for the diagnosis of the structure and their usefulness in a broader decision-making framework is discussed.
\end{abstract}

Keywords: Masonry, heritage, environmental effects, dynamic regression models, ARX, diagnosis

\section{INTRODUCTION}

The need to safeguard heritage structures is now widely accepted and a large number of the world's current stock of recognised heritage buildings are made of masonry. Since such structures are often affected by very slow deterioration mechanisms that are difficult to identify, structural health monitoring (SHM) has emerged as a powerful tool for th early identification of damage and to assess the effectiveness of repairs.

With respect to masonry heritage structures, SHM strategies can be classified as dynamic or static. Dynamic monitoring involves the identification and control of dynamic properties such as natural frequencies, mode shapes and damping ratios ${ }^{1,2}$ whereas static monitoring involves the continuous measurement of key slow-varying parameters over a long time period. ${ }^{3}$ Although dynamic SHM can prove to be extremely useful to correctly assess the vulnerability of structures to dynamic loads and to evaluate the effectiveness of repairs, static monitoring is particularly suitable for the early identification of very slow damage mechanisms characterised by slow evolution rates. For masonry heritage structures, crack widths and the inclination of structural members are typically the most useful parameters to monitor as part of a static SHM campaign. There exist many examples whereby

Further author information: (Send correspondence to N.M.)

N.M.: E-mail: nirvan.makoond@upc.edu, Telephone: +34 934011036

L.P.: E-mail: luca.pela@upc.edu, Telephone: +34 934011036

C.M.: E-mail: climent.molins@upc.edu, Telephone: +34 934011053

P.R.: E-mail: pere.roca@upc.edu, Telephone: +34 937398636 
static SHM has been employed to successfully identify deterioration mechanisms affecting masonry heritage structures. ${ }^{4-7}$ Many of these have relied on simply fitting linear or nonlinear models to the acquired time series of monitored structural responses to evaluate their evolutionary condition.

However, due to the slow evolution rates of deterioration mechanisms of interest, irreversible components of measurements tied to damage are often in the same magnitude range as reversible changes linked to environmental variations. This can make the task of identifying underlying evolution trends challenging. Moreover, since directly fitting selected models to acquired time series provide no direct information on the relationship between environmental and structural parameters, the interpretation of analysis results from such methods is difficult. A very simple yet effective approach to this problem involves filtering out the effect of environmental effects using an identified linear trend between monitored structural and environmental parameters. Since much of the reversible variation experienced by the structural response of masonry structures can be attributed to temperature fluctuations, the latter has emerged as one of the most effective predictors to be used for this type of analysis. However, the relationship between temperature and structural responses cannot always be well represented by simple linear trend lines since they cannot account for effects caused by thermal inertia or by differences between interior and exterior temperature. Recently, dynamic linear regression models, specifically those including an Auto-Regressive output and an eXogenous input (ARX), have proved to be an attractive solution for this problem since they are able to represent response variables when they depend linearly on their own rate of change, on the rate of change of predictors and on the present value of predictors. ${ }^{8}$ Furthermore, the application of such models has been facilitated by the recent proposal of a fully automated procedure for identifying irreversible components linked to active deterioration mechanisms from acquired time histories of monitored structural responses in masonry heritage structures. ${ }^{9}$ In addition to simplifying the implementation of ARX models, the method proposed in Ref. 9 also comprehends an automatic classification procedure to facilitate the interpretation of analysis results.

This paper demonstrates the implementation of the above-mentioned fully automated procedure to the case of a SHM system installed in a complex medieval heritage structure through a user-friendly tool developed in the MATLAB ${ }^{\circledR}$ environment. ${ }^{10}$ The tool can be used to update analysis results quickly and easily as new data is received from the monitoring system. The structure in question is the church of the Sant Cugat monastery close to Barcelona built mostly between the 12th and 15th centuries. Through this full-scale application, the advantages and drawbacks of implemented methods are also discussed.

First, a brief description of the health monitoring system is presented. All data processing steps and analysis methodologies implemented in the MATLAB ${ }^{\circledR}$ tool are then presented. The results from all the analysis methodologies are then compared leading to the general conclusions on the state of the structure and on the employed methodology in general.

\section{STRUCTURAL HEALTH MONITORING SYSTEM}

Several signs of damage, notably in the form of cracks and inclinations, can be observed on the medieval structure of the church of the monastery of Sant Cugat. Because some of these could be linked to active deterioration mechanisms that could pose a threat to the structural integrity of the church in the future, a long-term SHM system was installed in 2017 to better understand the true evolutionary nature of key structural responses.

After carrying out extensive damage surveys and analyses, 14 cracks and 2 key inclinations were identified as being the most suitable responses to be monitored as part of the SHM system. Relevant environmental parameters on-site were also chosen to be monitored as part of the system. These include interior and exterior temperature and relative humidity. Most of the sensors were installed in March 2017 with the exception of 3 crackmeters. Two of which were installed in December 2017 before the installation of the final sensor in April 2018. As summarised in Table 1, the complete static SHM system installed consists of 22 sensors. Since all analysis results presented in this paper utilise data collected up to 17/03/2020, the total duration for which monitoring data is available from each sensor is also reported in Table 1. The position of all the sensors as well as the layout of the monitoring system is shown in Figure 1. 
Table 1. Summary of sensors used in the structural health monitoring system installed in the monastery of Sant Cugat together with their location and the total duration of useful data collected up to 17/03/2020.

\begin{tabular}{cccc}
\hline Location & Sensor & Total duration of useful data [years] & Sensor type \\
\hline \multirow{2}{*}{ Fourth Nave } & FS-1.1 & 3.02 & Crackmeter \\
& FS-1.2 & 3.02 & Crackmeter \\
\hline Bell tower & INC-1.3 & 3.02 & Inclinometer \\
Bell tower (exterior) & TEMP-2.4 & 3.02 & Thermistor \\
& HUMI-2.4 & 3.02 & Humidity Sensor \\
\hline Sacristy & FS-2.5 & 3.02 & Crackmeter \\
\hline \multirow{5}{*}{ Lateral aisle(bell tower) } & FS-2.6 & 3.02 & Crackmeter \\
& FS-2.7 & 3.02 & Crackmeter \\
& TEMP-2.9 & 3.02 & Inclinometer \\
& HUMI-2.10 & 3.02 & Thermistor \\
Central nave & FS-2.11 & 3.02 & Humidity Sensor \\
\hline \multirow{2}{*}{ Central nave (exterior) } & FS-3.15 & 3.02 & Crackmeter \\
& FS-3.13 & 3.02 & Crackmeter \\
\hline \multirow{2}{*}{ Lateral aisle(monastery) } & TEMP-3.12 & 3.02 & Crackmeter \\
& FS-3.14 & 3.02 & Thermistor \\
& FS-3.17 & 3.02 & Humidity Sensor \\
Apse & FS-3.18 & 3.02 & Crackmeter \\
Linterior front façade & FS-3.19 & 3.02 & Crackmeter \\
\hline \multirow{2}{*}{ FS-3.20 } & 3.02 & Crackmeter \\
\hline
\end{tabular}

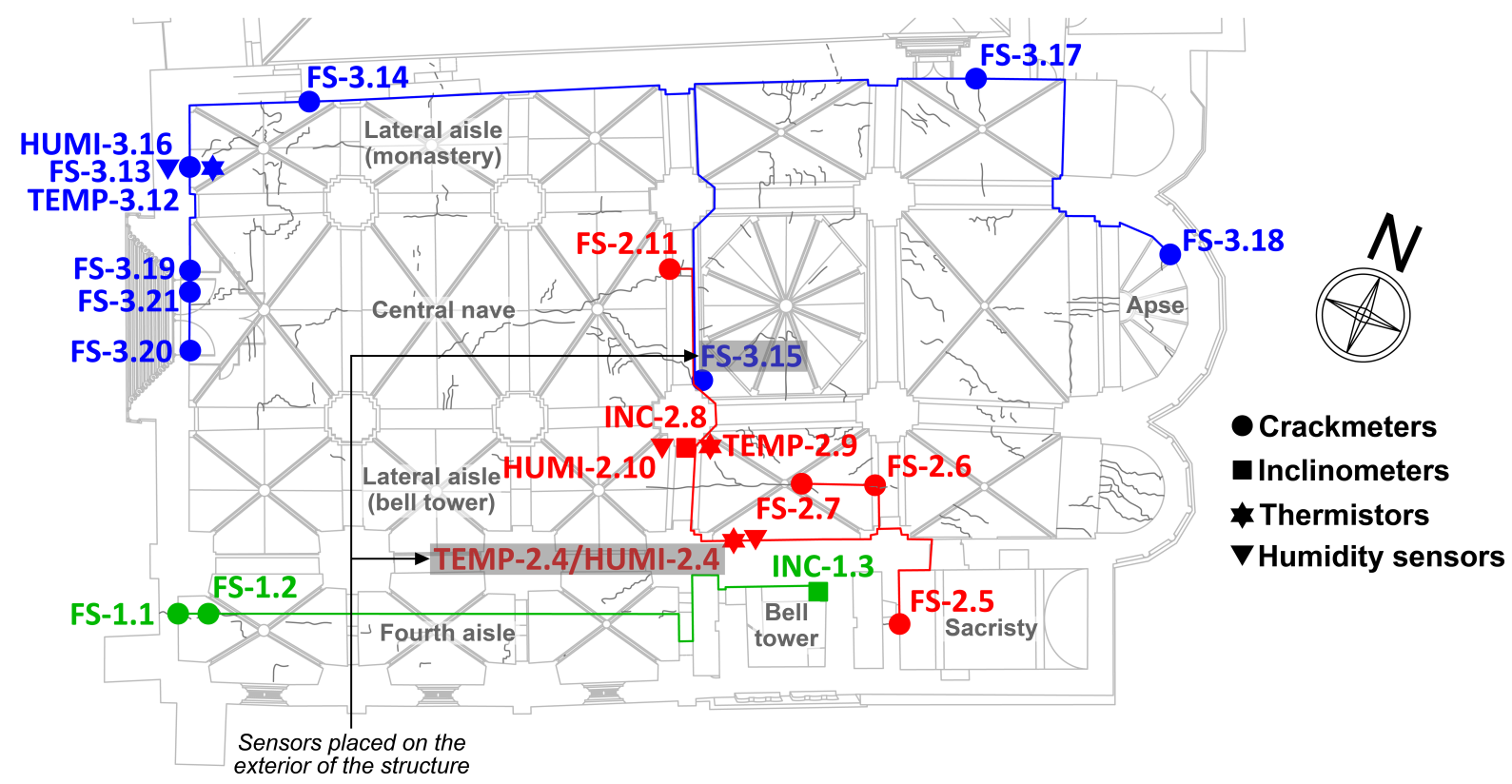

Figure 1. Plan view showing position of sensors and layout of structural health monitoring system. A different colour is assigned to each group of sensors connected to the same data logger or expansion module. 


\section{DATA PROCESSING AND ANALYSIS}

This section presents the processing steps and analysis methodologies applied to the raw data collected from the sensors installed in the monastery of Sant Cugat.

\subsection{Data pre-processing}

When it comes to static SHM of masonry heritage structures, two stages of pre-processing are normally implemented before any further analysis is carried out. Since raw signals from sensors come in a variety of forms such as voltage, current, resistance or frequency, the first pre-processing stage consists in converting these signals to meaningful physical units as well as correcting the readings to account for thermal expansion of the materials making up the sensors. It is convenient to automatically program this pre-processing stage at the level of the data logger itself. The second pre-processing stage involves removing any anomalies present in the data that are not caused by a physical phenomena related to the structural behaviour. These often appear as "spikes" in sensor data ${ }^{11}$ and can originate from several sources such as capacitive or inductive noise in the analog signal path, communication errors ${ }^{12}$ or undesired external interactions with the sensor. With respect to the SHM system installed in the monastery of Sant Cugat, on rare occasions where such spikes were identified, the data points related to the anomaly were deleted and replaced with corresponding values based on linear interpolation of neighbouring non-anomalous values. This was considered an acceptable approach for the case of static SHM because it can be expected that anomalies will be scarce due to the high quality of modern sensors and cables, combined with the comparatively low sampling rate of such systems (samples usually collected every 15 min or every hour). Moreover, any complex procedure to detect and remove anomalies will incur an additional risk of eliminating data that could provide valuable information on the evolution of the response variables in question. Nevertheless, it must be said that since anomaly detection from time series is an important problem with applications in many fields, ${ }^{13}$ there exist several techniques that can be applied to automate their identification and removal, including some that have been used for other types of SHM systems. ${ }^{11,12}$

\subsection{Linear regression of time series}

The simplest method applied to the analysis of data from the static SHM system in Sant Cugat monastery involves fitting a linear trend line to the evolution of the data from each structural response as shown in Figure 2. The slope of the identified trend line is then used as an estimate of the evolution rate of the structural response.

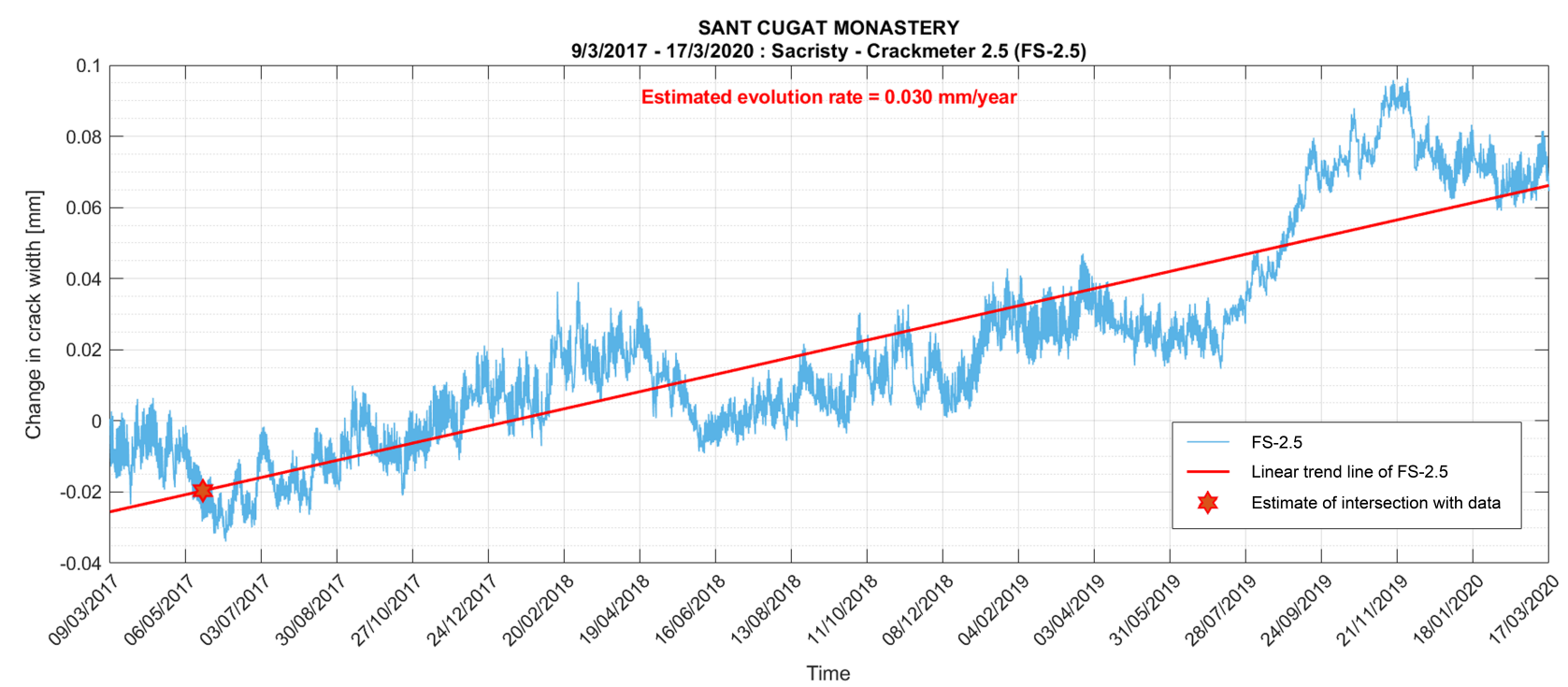

Figure 2. Linear regression of time series.

Although this method has been used successfully in the past, particularly when very long periods of monitoring data are available, it can easily be influenced by irregular fluctuations which are common in this type of data. 
Moreover, because it attempts to represent a clearly nonlinear progression using a linear model, there are no reasonable ways to assess the goodness of the fit.

\subsection{Nonlinear regression of time series (periodic model)}

As shown in Figure 3, a more sophisticated approach compared to the one presented in Section 3.2 involves directly fitting the time series of each monitored response to the nonlinear periodic function shown in Equation (1) which includes a periodic component $(A \sin (P \boldsymbol{t}-\phi))$ and a linear one $(B \boldsymbol{t}+C)$. The evaluated value of $B$ from Equation (1) after the fitting procedure is then used as an estimate of the evolution rate of the structural response.

$$
y=A \sin (P \boldsymbol{t}-\phi)+B \boldsymbol{t}+C
$$

where $y$ is the monitored structural parameter of interest, $t$ is time while $A, P, \phi, B$ and $C$ are unknowns that can be found using the Levenberg-Marquardt algorithm. ${ }^{14} A, P$ and $\frac{\phi}{P}$ correspond respectively to the amplitude, period and phase shift of the periodic component of the function while $B$ and $C$ correspond respectively to the slope and y-intercept of the straight line component.

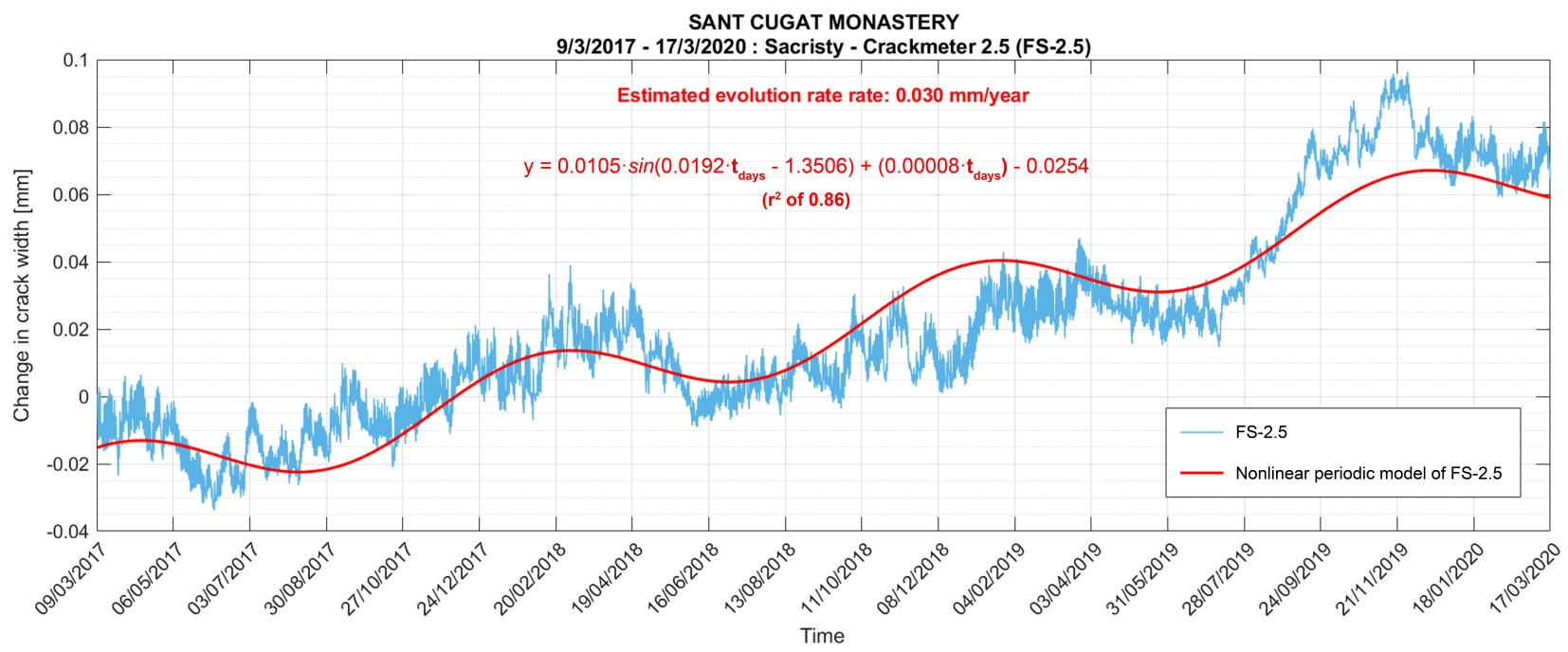

Figure 3. Nonlinear periodic model fitted to time series.

Because this method attempts to model the actual nonlinear behaviour of the response, the coefficient of determination $\left(r^{2}\right)$ can be used to assess the quality of the fit. This metric varies between 0 and 1 with values closer to 1 indicating a better correlation. ${ }^{15}$ Because seasonal cycles are known to have a period corresponding to the duration of a tropical year, the closeness of the identified period to this duration can also be used to assess the suitability of the fit. As recommended in Ref.9, estimated evolution rates are discarded if $\left(r^{2}\right)$ is less than 0.6 and if the identified period differs from the duration of a tropical year by more than $25 \%$.

Although this method provides an improvement from simple linear regression of the time series of monitored responses, reversible effects caused by changes in environmental parameters cannot always be well represented by a periodic function. Because it fails to directly consider any monitored environmental parameters, this model cannot account for such effects which can sometimes lead to a misrepresentation of the actual evolutionary condition of monitored structural responses.

\subsection{Preliminary evaluation of correlation with temperature}

Before implementing any analysis methodology which takes advantage of actual records of environmental parameters monitored on-site, a preliminary analysis needs to be carried out in order to identify which environmental parameters have the greatest influence on the evolution of monitored structural responses. This can be achieved 
using the Pearson correlation coefficient ${ }^{15}$ between monitored environmental and structural parameters $\left(R_{X, Y}\right)$. As described by Equation (2), this coefficient between two random variables $X$ and $Y$, with standard deviations of $\sigma_{X}$ and $\sigma_{Y}$ respectively, can be understood as a dimensionless measure of their linear dependence.

$$
R_{X, Y}=\frac{\operatorname{cov}(X, Y)}{\sigma_{X} \cdot \sigma_{Y}}
$$

$R_{X, Y}$ can vary between -1 and +1 with absolute values closer to unity indicating a better correlation. The sign of the coefficient indicates the type of correlation. A negative sign means that an increase of one parameter leads to a decrease of the other while a positive one signals the opposite.

Using this coefficient, a preliminary evaluation of correlation with internal and external temperature and relative humidity was carried out for each structural response monitored in the monastery of Sant Cugat. In general, the correlation with temperatures was found to be significantly stronger.

\subsection{Filtering environmental effects through linear regression}

The simplest implemented analysis method which explicitly makes use of monitored environmental parameters involves simply carrying out a linear regression between each structural response and either the internal or external monitored temperature depending on which one has the greatest correlation coefficient described in Section 3.4. Once these linear models are identified, they are used to simulate the variations of each structural response caused by changes in temperature. As shown in Figure 4, the residuals found by subtracting the estimated temperature effects from actual measurements is then used as an estimate of the underlying changes exhibited by the structural response outside reversible ones caused by changing environmental effects. An estimate of the evolution rate is then obtain by performing a simple regression of these filtered residuals.

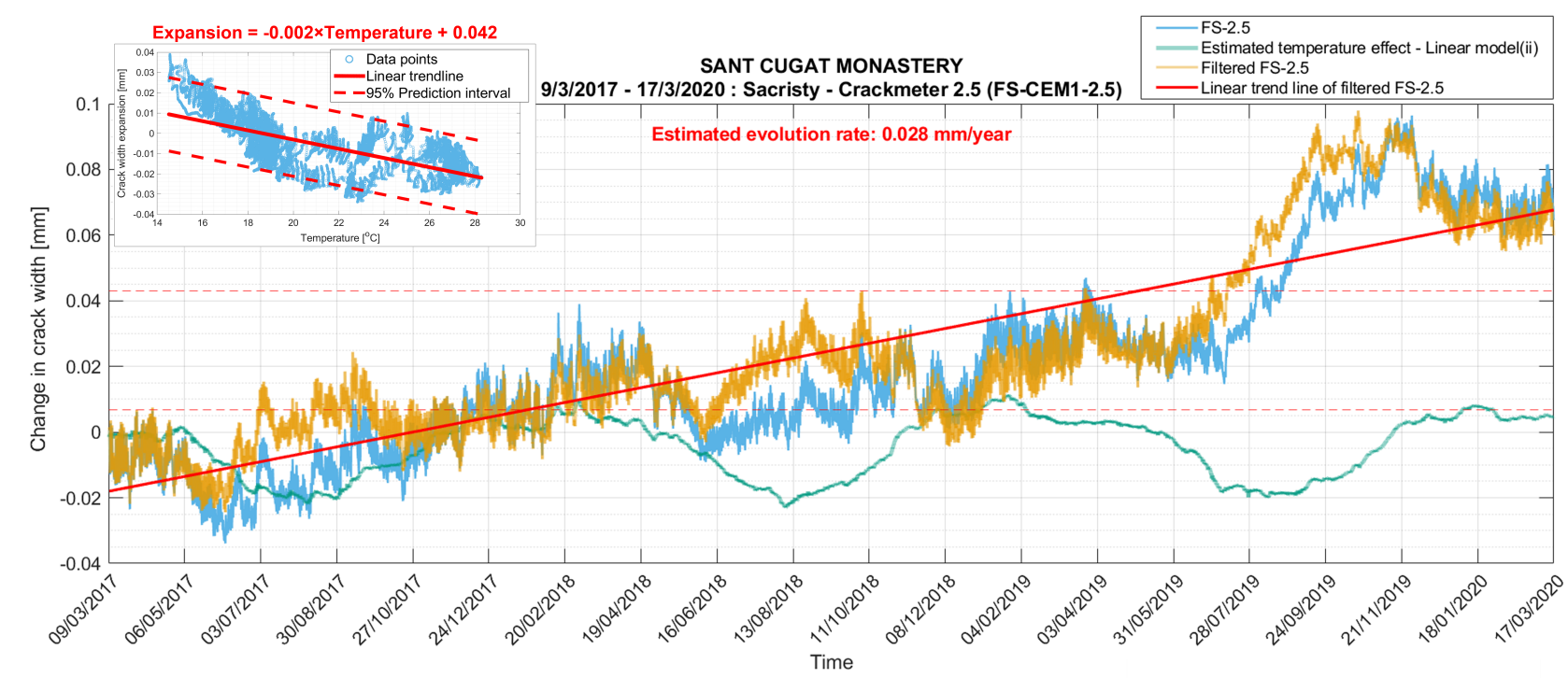

Figure 4. Filtering of temperature effect based on identified general linear trend. The regression with measured temperature values used to estimate the effect of temperature on crack width expansion is also shown.

In order for the linear models to be able to encompass most of the reversible variations typically experienced by a structural response during a full seasonal cycle, the data from at least a complete year of monitoring needs to be used during the initial regression procedure with temperature. Although using data from more than a year could risk incorporating effects linked to deterioration mechanisms in the simple linear models, it could also lead to an improved representation of the evolving relationship between temperature and structural responses. As such, two sets of linear regression are performed for each pair of monitored structural responses and temperature 
as described below. The results from both these methods are then compared and used in the classification procedure presented in Section 3.7.

- Linear model(i): Linear regression between temperature and structural response using data from the entire monitoring period.

- Linear model(ii): Linear regression between temperature and structural response only using data from a full calender year (hereafter referred to as the estimation phase).

The fact that this method makes use of actual measurements of temperature to evaluate its effect means that it is able to better account for reversible changes experienced by structural parameters in comparison to simply assuming a uniform periodic behaviour in the absence of deterioration mechanisms. However, although it provides a sufficiently accurate representation for many cases, a simple linear model is certainly an oversimplification of the actual relationship between temperature and monitored structural parameters. Specifically, since each measured value of the response is only compared to a single value of temperature corresponding to the same point in time during the regression process, such models cannot account for effects caused by thermal inertia or by thermal gradients between interior and exterior temperatures.

\subsection{Filtering environmental effects with ARX models}

The most sophisticated analysis methodology used to estimate the filtered evolution rate of monitored structural responses as part of the SHM system installed in the monastery of Sant Cugat makes use of Auto-Regressive models incorporating an eXogenous input (ARX). Such models are better equipped to capture the actual system dynamics of the relationship between temperature and structural parameters because they use measured values of past responses together with those of past and current or delayed predictors to represent the current value of the response. In an initial stage, QR factorization ${ }^{16}$ was used to identify the most suitable coefficients of the ARX models using data collected over the estimation phase. These identified models were then incorporated in the developed MATLAB ${ }^{\circledR}$ tool so that the changes of each monitored structural parameter caused by temperature variations can be simulated from measured predictors with data collected over the entire monitoring period (simulation phase). As was the case for the method presented in Section 3.5, the simulated temperature effect is then subtracted from the actual measurements of each structural response and its evolution rate is then estimated using regression of the filtered residuals.

In this case, two types of ARX models have been employed for the analysis. Firstly, a Single-Input-SingleOutput (SISO) ARX model was identified incorporating the same predictor as the linear models described in Section 3.5. Secondly, a Multiple-Input-Single-Output (MISO) ARX model was identified incorporating both interior and exterior temperatures as predictors. Although it is expected that some thermal inertia effects can be captured by both models, the MISO models are expected to better capture the effects caused by thermal gradients between interior and exterior temperature.

The detailed procedure incorporating ARX models used for the analysis of data from the static SHM system in the monastery of Sant Cugat is described in Ref. 9 and summarised in Figure 5. As shown, besides the measurements of the response and of selected predictors acquired by sensors from the SHM system, the only other required input to the procedure is the range of model orders to be tested. In this case, a range corresponding to a duration from 5 to 25 days was specified for the SISO ARX models, and one corresponding to a duration from 4 to 10 days was specified for the MISO ARX models.

It is worth mentioning that for the 16 structural responses analysed from the SHM system in the monastery of Sant Cugat, the $95 \%$ prediction interval associated to ARX models between temperature and structural responses were on average $13 \%$ smaller when compared to those associated to the linear models. Specifically, the prediction intervals of ARX models were smaller than those of the linear ones for 13 out of the 16 monitored responses with 6 sensors showing an improvement of more than 20\%. This clearly shows the greater suitability of ARX models to represent this relationship. 


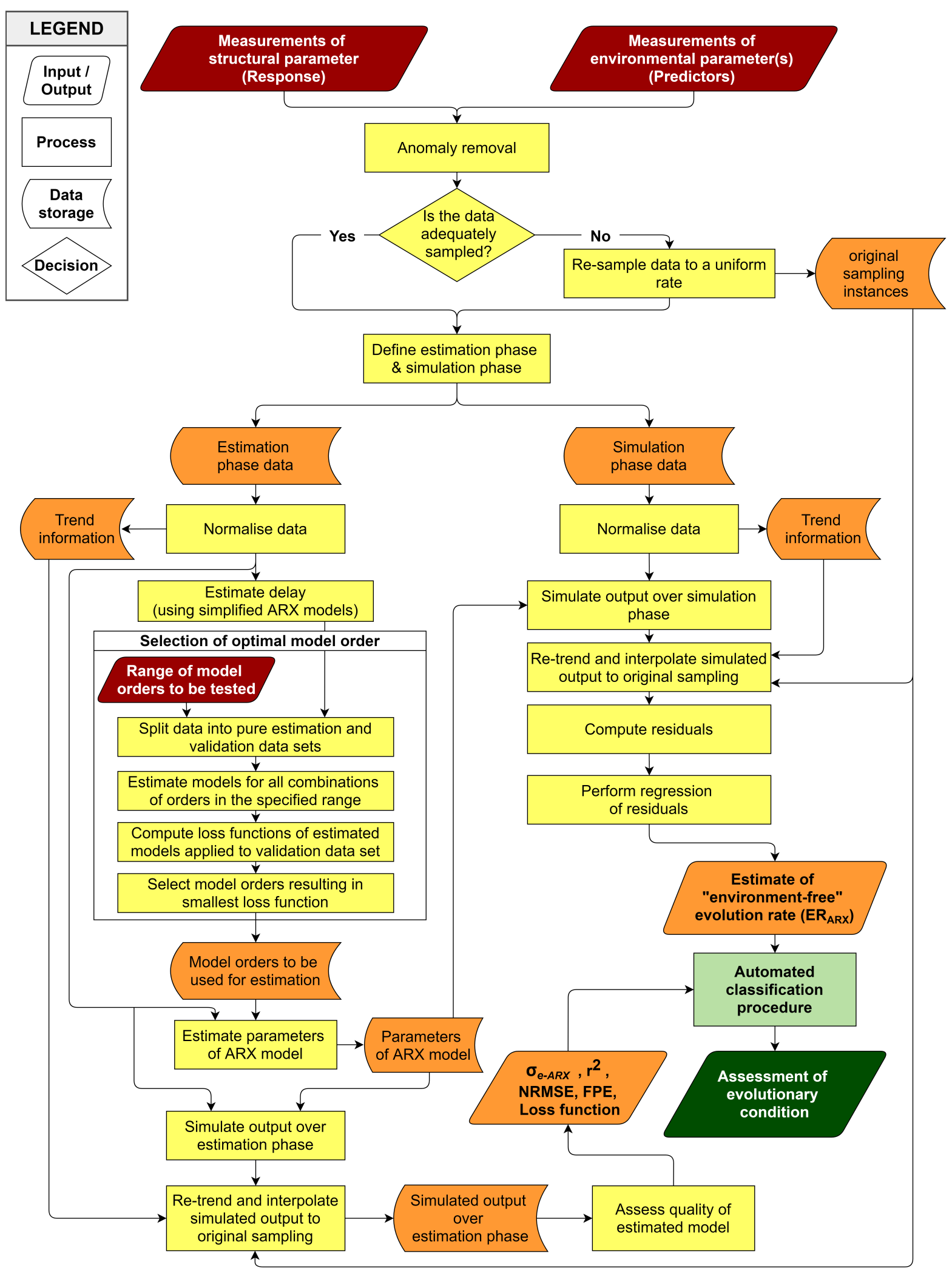

Figure 5. Procedure for the implementation of ARX models to filter out the effect of measured environmental parameters and assess the evolutionary condition of a monitored structural parameter. 


\subsection{Automated classification procedure}

Although using ARX models to filter out the reversible temperature effects from monitored structural responses can greatly improve the accuracy of their estimated underlying evolution rates, it is still important to consider the errors associated to the models when interpreting the results. The automated classification procedure proposed in Ref. 9 can greatly facilitate this task. The procedure relies on a series of 5 sequentially applied tests (summarised in Figure 6), all of which rely on two key values evaluated for each sensor. The first of which is the filtered evolution rate estimated using the most extensive ARX model employed $\left(E R_{A R X}\right)$. The second key value is the standard error of the estimate computed as shown in Equation (3) from the residuals over the estimation phase associated to the same ARX model $\left(\sigma_{e-A R X}\right)$.

$$
\sigma_{e-A R X}=\sqrt{\frac{\sum_{i=1}^{n}\left(y_{i}-\hat{y}_{i}\right)^{2}}{n-k-1}}
$$

where $y_{i}$ refer to actual measurements of structural responses while $\hat{y}_{i}$ refer to model predictions. With $n$ equal to the number of data sample points and $k$ equal to the number of explanatory variables used in the model, $n-k-1$ represents the number of degrees of freedom available for the computation of the error metric.

Based on the outcomes of the 5 sequential tests, each monitored structural parameter is classified in one of the following 5 categories.

1. Stationary: Structural parameters showing a clear stationary trend outside reversible variations caused by environmental parameters.

2. Evolutionary: Structural parameters showing a clear evolutionary trend outside reversible variations caused by environmental parameters.

3. Apparently stationary: Structural parameters showing a stationary trend but for which there still is a rather large uncertainty associated to the estimation of the trend.

4. Apparently evolutionary: Structural parameters showing an evolutionary trend but for which there still is a rather large uncertainty associated to the estimation of the trend.

5. Inconclusive: Structural parameters for which no clear conclusion can be made on its evolutionary state from the available monitoring data alone.

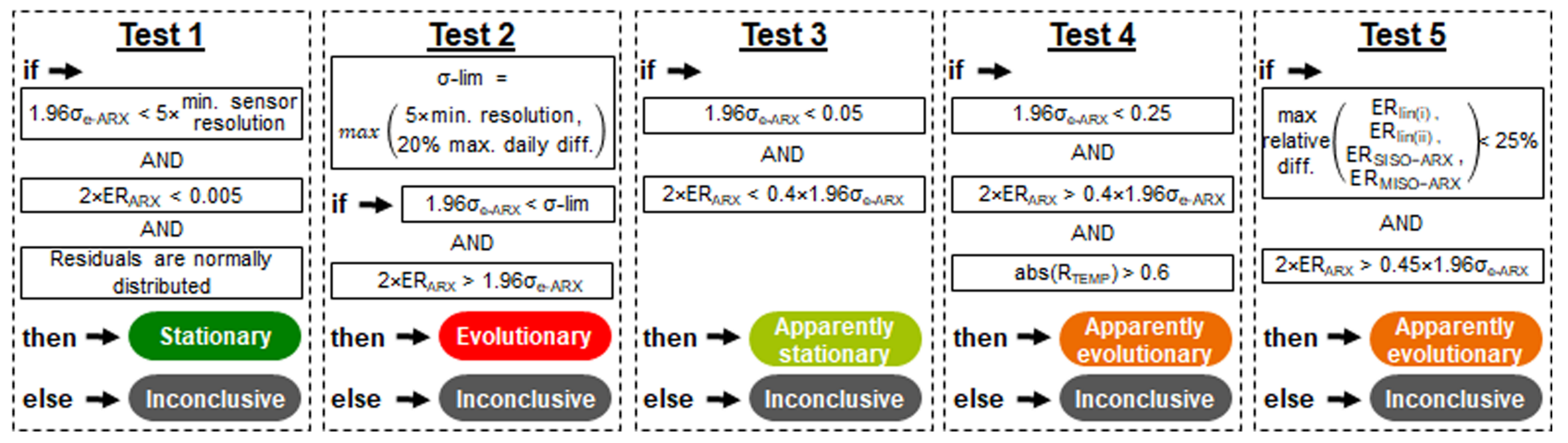

Figure 6. Summary of tests used for the classification procedure of monitored structural responses. 


\section{TOOL FOR PERIODIC UPDATING OF ANALYSIS}

All the processing steps and analysis methodologies described in Section 3 have been implemented in an easyto-use MATLAB ${ }^{\circledR}$ tool that can be used to download files containing updated data from the sensors installed in the monastery of Sant Cugat. These files are uploaded daily from the data acquisition system in place on-site via a wireless network to a remote server from which the MATLAB ${ }^{\circledR}$ tool can download the files.

The tool consists of two interfaces which the user can interact with to carry out several tasks. The Main window which appears upon running the application is shown in Figure 7.

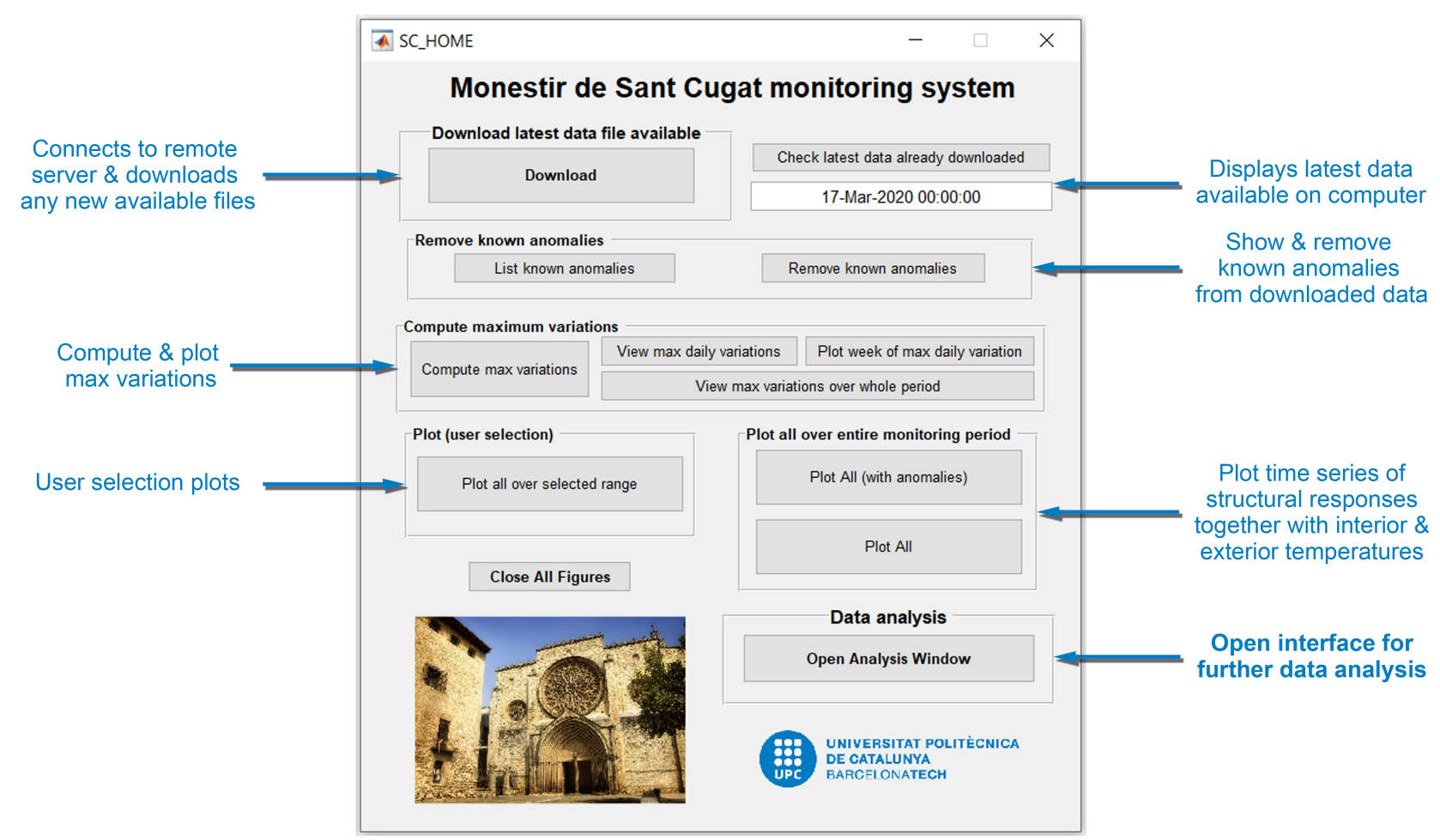

Figure 7. Main window of the application used to carry out periodic verifications of data from the monitoring system installed in the monastery of Sant Cugat.

From the Main window, the user can verify the latest data stored on the user's system, download any new data available from the remote server, remove known anomalies from the data, compute maximum variations of each response which can be used for comparison to established thresholds and create different plots showing the evolution of the acquired data.

The Analysis window shown in Figure 8 can also be launched from the Main window. From this window, all the analysis procedures described from Sections 3.2 to 3.7 can be executed and the user can then choose to extract or visualise any updated results within minutes. 


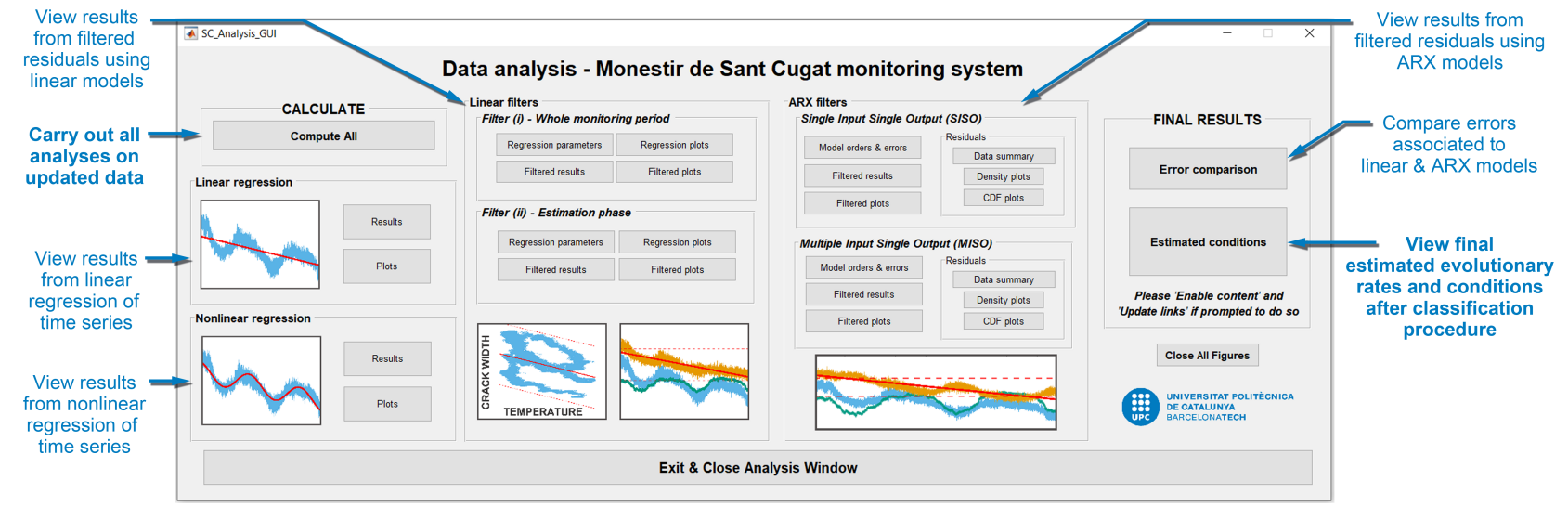

Figure 8. Analysis interface of the application used to carry out periodic verifications of data from the monitoring system installed in the monastery of Sant Cugat.

\section{ANALYSIS RESULTS}

The predicted evolution rates for all monitored structural parameters in the monastery of Sant Cugat from methods based on directly fitting time series to selected models are shown in Table 2.

Table 2. Comparison of estimated evolution rates for monitored structural parameters from methods based on directly fitting time series to selected models. (Based on data up to $17 / 03 / 2020$ )

\begin{tabular}{|c|c|c|c|}
\hline \multirow[b]{2}{*}{ Sensor } & \multirow[b]{2}{*}{ unit } & \multicolumn{2}{|c|}{ Estimate of annual evolution rate [unit/year } \\
\hline & & Linear regression & Nonlinear regression \\
\hline FS-1.1 & $\mathrm{mm}$ & 0.108 & 0.095 \\
\hline FS-1.2 & $\mathrm{mm}$ & 0.000 & 0.003 \\
\hline INC-1.3 & $\circ$ & -0.002 & -0.003 \\
\hline FS-2.5 & $\mathrm{mm}$ & 0.030 & 0.030 \\
\hline FS-2.6 & $\mathrm{mm}$ & -0.001 & -0.001 \\
\hline FS-2.7 & $\mathrm{mm}$ & 0.001 & 0.001 \\
\hline INC-2.8 & $\circ$ & -0.005 & -0.006 \\
\hline FS-2.11 & $\mathrm{mm}$ & 0.082 & 0.055 \\
\hline FS-3.15 & $\mathrm{mm}$ & 0.001 & - \\
\hline FS-3.13 & $\mathrm{mm}$ & 0.015 & -0.008 \\
\hline FS-3.14 & $\mathrm{mm}$ & -0.006 & -0.018 \\
\hline FS-3.17 & $\mathrm{mm}$ & -0.038 & -0.046 \\
\hline FS-3.18 & $\mathrm{mm}$ & -0.001 & 0.007 \\
\hline FS-3.19 & $\mathrm{mm}$ & -0.099 & -0.077 \\
\hline FS-3.20 & $\mathrm{mm}$ & -0.037 & -0.038 \\
\hline FS-3.21 & $\mathrm{mm}$ & 0.182 & 0.111 \\
\hline
\end{tabular}

The evolution rate predicted for crackmeter FS-3.15 was disregarded because it had a poor correlation with the data (coefficient of determination of 0.41) indicating that the evolution of the data could not be well represented by the nonlinear periodic model. Despite the greater complexity of the nonlinear model, it is clear that the estimated evolution rates between the two methods are in good agreement for certain cases, notably for 
crackmeters FS-2.5, FS-2.6 and FS-2.7 and for inclinometer INC-2.8.

The evolution rates estimated after filtering out simulated environmental effects using linear and ARX models are presented in Table 3 together with the estimated condition of each response using the procedure described in Section 3.7.

Table 3. Estimated evolution rates of monitored structural parameters from methods filtering out the simulated effect of temperature and estimated condition from automated classification procedure. (Based on data up to 17/03/2020)

\begin{tabular}{ccccccc}
\hline & & \multicolumn{2}{c}{ Estimate of annual evolution rate [unit/year] } & Estimated condition \\
\cline { 3 - 6 } Sensor & unit & $\begin{array}{c}\text { Linear filter } \\
(\mathbf{i})\end{array}$ & $\begin{array}{c}\text { Linear filter } \\
\text { (ii) }\end{array}$ & $\begin{array}{c}\text { SISO } \\
\text { ARX }\end{array}$ & $\begin{array}{c}\text { MISO } \\
\text { ARX }\end{array}$ & \\
\hline FS-1.1 & $\mathrm{mm}$ & 0.100 & 0.101 & 0.098 & 0.100 & App. Evolutionary \\
FS-1.2 & $\mathrm{mm}$ & 0.002 & 0.002 & 0.003 & 0.003 & App. Evolutionary \\
\hline INC-1.3 & $\mathbf{\circ}$ & -0.003 & -0.003 & -0.003 & -0.003 & Evolutionary \\
\hline FS-2.5 & $\mathrm{mm}$ & 0.028 & 0.028 & 0.025 & 0.026 & Evolutionary \\
\hline FS-2.6 & $\mathrm{mm}$ & 0.001 & 0.000 & 0.000 & 0.000 & App. Stationary \\
FS-2.7 & $\mathrm{mm}$ & 0.001 & 0.001 & 0.001 & 0.002 & Stationary \\
INC-2.8 & $\circ$ & -0.006 & -0.006 & -0.006 & -0.007 & Evolutionary \\
\hline FS-2.11 & $\mathrm{mm}$ & 0.066 & 0.064 & 0.069 & 0.066 & App. Evolutionary \\
FS-3.15 & $\mathrm{mm}$ & -0.004 & -0.005 & -0.006 & -0.005 & Inconclusive \\
\hline FS-3.13 & $\mathrm{mm}$ & -0.001 & 0.000 & -0.003 & -0.005 & App. Stationary \\
FS-3.14 & $\mathrm{mm}$ & -0.012 & -0.014 & -0.013 & -0.014 & Inconclusive \\
FS-3.17 & $\mathrm{mm}$ & -0.041 & -0.042 & -0.041 & -0.048 & App. Evolutionary \\
\hline FS-3.18 & $\mathrm{mm}$ & 0.003 & 0.004 & 0.001 & 0.008 & App. Evolutionary \\
\hline FS-3.19 & $\mathrm{mm}$ & -0.093 & -0.093 & -0.110 & -0.080 & App. Evolutionary \\
FS-3.20 & $\mathrm{mm}$ & -0.033 & -0.032 & -0.037 & -0.019 & Inconclusive \\
FS-3.21 & $\mathrm{mm}$ & 0.119 & 0.116 & 0.141 & 0.099 & App. Evolutionary \\
\hline
\end{tabular}

Based on the outcomes of the classification procedure, the most definitive conclusions that can be made from the data collected up to the 17th of March 2020 relate to the responses being monitored by FS-2.7, FS-2.5, INC-1.3 and INC-2.8. It appears that the crack monitored by sensor FS-2.7 is stationary outside seasonal fluctuations. On the other hand, the crack monitored by FS-2.5 shows an underlying opening trend of approximately $0.026 \mathrm{~mm} /$ year while the bell tower (monitored by INC-1.3) and the pillar in the south-west corner under the cimborio (monitored by INC-2.8) seem to be leaning towards the south-east with inclination rates of $0.003^{\circ} / \mathrm{year}^{\circ}$ and $0.007^{\circ} /$ year respectively. It is interesting to note that the predictions of simpler analysis methods based simply on fitting selected models to acquired time series are also in good agreement with trends predicted from filtered residuals for these specific sensors.

\section{CONCLUSIONS}

One of the main conclusions that can be derived from the identified trends following the analyses presented in this paper is that the structure of the church of the monastery of Sant Cugat is most likely affected by a deterioration mechanism linked to the outward rotation of the bell tower towards the south-east as shown in Figure 9(a). The opening trend observed for the crack in the sacristy is in good agreement with this hypothesis because the sacristy is intrinsically tied to the bell tower. In fact, it indicates that the center of rotation linked to the movement of the bell tower most probably lies at a rather low point of the structure. Following initial analyses from the SHM system, recent topographic surveys reveal that the southern and eastern walls of the bell tower actually have inclinations of up to $1.5 \%$ with a net displacement of $52 \mathrm{~cm}$ from the vertical position at 
the top of the main body of the tower ${ }^{17}$ as shown in Figure 9(b). Assuming rigid body motion, the evolutionary trend of $0.003 \%$ year estimated from the analysis of the SHM data would correspond to a further increase of $1.5 \mathrm{~cm}$ every 10 years of the net displacement at the top of the main body of the tower. This clearly suggests that an intervention could be required in the foreseeable future to prevent the deterioration mechanism causing these movements from impairing the structural integrity of this heritage structure. The analysis of the SHM data also revealed that other parts of the structure could be affected by this mechanism as one of the pillars supporting the cimborio exhibited an outward inclining trend in the same direction as that of the bell tower as shown in Figure 9(a).

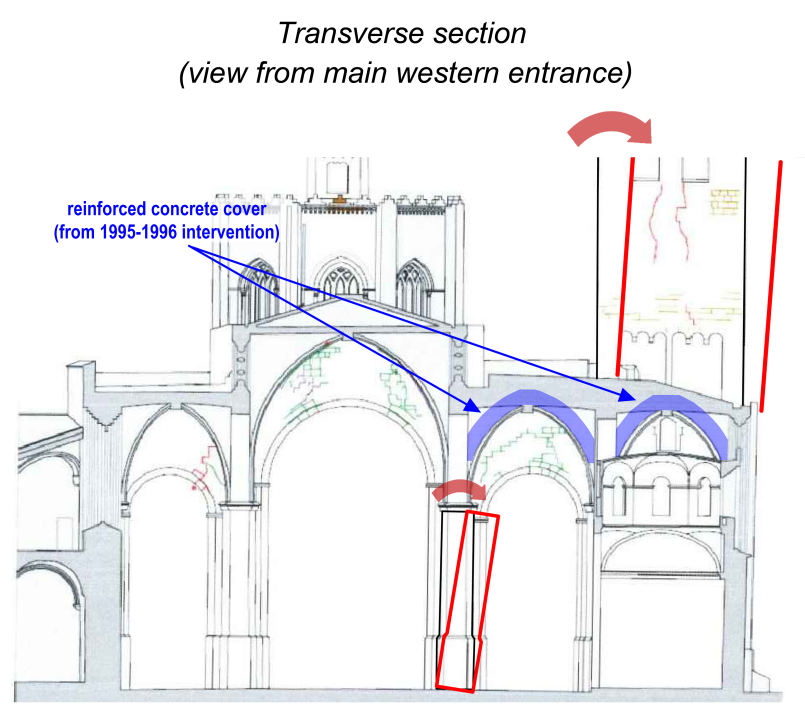

(a)

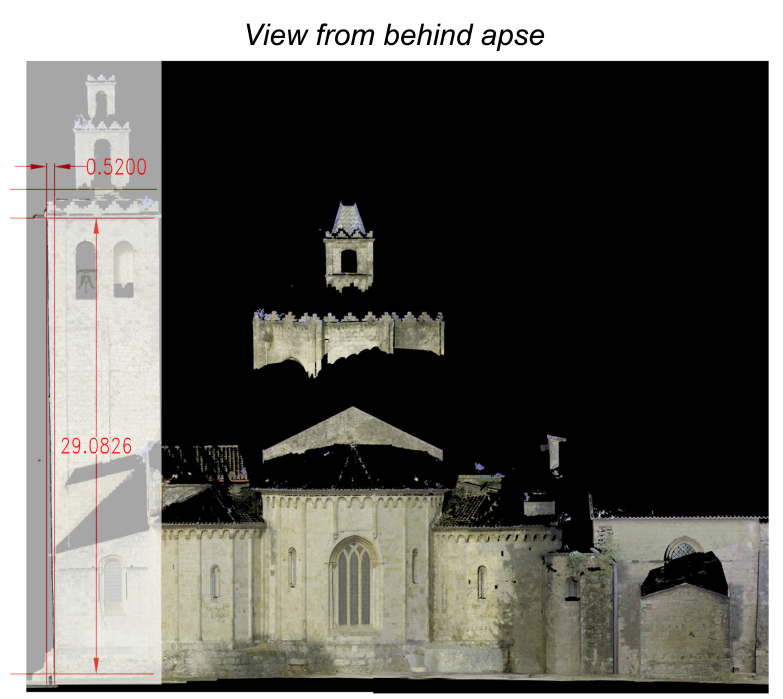

(b)

Figure 9. (a) Observed inclination trends from static monitoring campaign. (b) Measured inclination of the bell tower of Sant Cugat (all measurements shown in $\mathrm{m}) .{ }^{17}$

The analysis of the SHM data also revealed the effectively stationary trend of the monitored crack across the vault of the lateral aisle between the cimborio and the bell tower. Although this can partially be attributed to the increased ability of vaults to deform without suffering irreversible damage in comparison to stiffer members, it could also be linked to some strengthening measures carried out towards the end of the 20th century. In addition to inserting several rods in an attempt to stitch southern parts of the structure to the main body of the church, the intervention also involved placing a reinforced concrete cover over some of the vaults on the southern side of the structure as shown in Figure 9.

The case study presented in this paper clearly demonstrates the usefulness of static SHM for the diagnosis of heritage structures. It also reveals how the correct implementation of computational tools for processing SHM data can facilitate the task of extracting meaningful conclusions with respect to the diagnosis of heritage structures. When combined with adequate analysis procedures, SHM systems can provide quantitative evidence that can prove invaluable when making decisions on the need for structural interventions. In fact, outcomes of SHM analysis do not only provide information on which parts of the structure are vulnerable but also on deterioration rates. These can be used as key quantitative indicators to assess the urgency of different possible instability scenarios thus facilitating the task of prioritising diagnostic or intervention activities leading to more efficient infrastructure management.

\section{ACKNOWLEDGMENTS}

This research has received financial support from the City Council of Sant Cugat (Ajuntament de Sant Cugat) through a project aimed at monitoring the structure of the church of the monastery of Sant Cugat (ref. num. 
C-10764). The authors also gratefully acknowledge the financial support from the Ministry of Science, Innovation and Universities of the Spanish Government (MCIU), the State Agency of Research (AEI), as well as the ERDF (European Regional Development Fund), through the SEVERUS project (Multilevel evaluation of seismic vulnerability and risk mitigation of masonry buildings in resilient historical urban centres, ref. num. RTI2018099589-B-I00). Support from the AGAUR agency of the Generalitat de Catalunya and the European Social Fund, in the form of a pre-doctoral grant awarded to the corresponding author is also gratefully acknowledged.

\section{REFERENCES}

[1] Foti, D., Ivorra, S., and Sabbà, M. F., "Dynamic Investigation of an Ancient Masonry Bell Tower with Operational Modal Analysis - A Non-Destructive Experimental Technique to Obtain the Dynamic Characteristics of a Structure," The Open Construction and Building Technology Journal 6, 384-391 (nov 2012).

[2] Ramos, L., Marques, L., Lourenço, P., De Roeck, G., Campos-Costa, A., and Roque, J., "Monitoring historical masonry structures with operational modal analysis: Two case studies," Mechanical Systems and Signal Processing 24, 1291-1305 (jul 2010).

[3] Lorenzoni, F., Integrated methodologies based on Structural Health Monitoring for the protection of Cutural Heritage buildings, phd thesis, University of Trento (2013).

[4] Ottoni, F. and Blasi, C., "Results of a 60-Year Monitoring System for Santa Maria del Fiore Dome in Florence," International Journal of Architectural Heritage 9, 7-24 (jan 2015).

[5] Sánchez, A. R., Meli, R., and Chávez, M. M., "Structural Monitoring of the Mexico City Cathedral (19902014)," International Journal of Architectural Heritage 10, 15583058.2015.1113332 (dec 2015).

[6] Baraccani, S., Silvestri, S., Gasparini, G., Palermo, M., Trombetti, T., Silvestri, E., Lancellotta, R., and Capra, A., "A Structural Analysis of the Modena Cathedral," International Journal of Architectural Heritage 10, 15583058.2015.1113344 (dec 2015).

[7] Masciotta, M.-G., Roque, J. C., Ramos, L. F., and Lourenço, P. B., "A multidisciplinary approach to assess the health state of heritage structures: The case study of the Church of Monastery of Jerónimos in Lisbon," Construction and Building Materials 116, 169-187 (jul 2016).

[8] Lorenzoni, F., Casarin, F., Caldon, M., Islami, K., and Modena, C., "Uncertainty quantification in structural health monitoring: Applications on cultural heritage buildings," Mechanical Systems and Signal Processing 66-67, 268-281 (jan 2016).

[9] Makoond, N., Pelà, L., Molins, C., Roca, P., and Alarcón, D., "Automated data analysis for static structural health monitoring of masonry heritage structures," Manuscript submitted for publication (2019).

[10] MathWorks, "MATLAB R2018b Documentation," (2018).

[11] Cornelis, B. and Peeters, B., "Online Bayesian spike removal algorithms for structural health monitoring of vehicle components," in [Eurodyn 2014: Proceedings of the 9th International Conference on Structural Dynamics], 2295-2301 (jul 2014).

[12] Halfpenny, A., "Data Preprocessing for Damage Detection," in [Encyclopedia of Structural Health Monitoring], John Wiley \& Sons, Ltd, Chichester, UK (jan 2008).

[13] Chakraborty, G., Kamiyama, T., Takahashi, H., and Kinoshita, T., "An Efficient Anomaly Detection in Quasi-Periodic Time Series Data-A Case Study with ECG," in [Time Series Analysis and Forecasting. ITISE 2017. Contributions to Statistics], 147-157, Springer, Cham (sep 2018).

[14] Moré, J. J., "The Levenberg-Marquardt algorithm: Implementation and theory," in [Numerical Analysis], Watson, G. A., ed., 105-116, Springer, Berlin, Heidelberg (1978).

[15] Asuero, A. G., Sayago, A., and González, A. G., "The Correlation Coefficient: An Overview," Critical Reviews in Analytical Chemistry 36, 41-59 (jan 2006).

[16] Björck, Å., "Solving linear least squares problems by Gram-Schmidt orthogonalization," BIT 7, 1-21 (mar 1967).

[17] Ajuntament de Sant Cugat (Sant Cugat City Council), "Informe tècnic: Comprovació de la verticalitat de la torre del campanar del monestir (Measurement of the inclination of the bell tower of the monastery)," tech. rep. (2019). 